\title{
Effect of Choline on the Morphology, Growth and Phospholipid Composition of Fusarium graminearum
}

\author{
By MARILYN G. WIEBE, * GEOFFREY D. ROBSON AND \\ ANTHONY P. J. TRINCI \\ Microbiology Group, Department of Cell and Structural Biology, School of Biological Sciences, \\ Stopford Building, University of Manchester, Manchester M13 9PT, UK
}

(Received 22 March 1989; revised 10 May 1989; accepted 17 May 1989)

\begin{abstract}
Studies were made of the growth kinetics, morphology and phospholipid composition of two strains of Fusarium graminearum, a wild-type strain (A3/5) and a highly branched variant (C106) which arose spontaneously during cultivation of A3/5. No significant difference was observed between the hyphal diameters of the two strains and therefore increased branching of $\mathrm{C} 106$ could not be explained in the terms of an increase in hyphal radius in the absence of a change in hyphal growth unit volume. The two strains had the same specific growth rate in batch culture and this was not affected by the addition of up to $1.5 \mathrm{~mm}$-choline to the medium. However, choline increased the mean hyphal extension rate and colony radial growth rate of both strains and this response was correlated with the formation of mycelia which were more sparsely branched than mycelia grown on medium lacking choline. Addition of betaine, choline, ethanolamine, monomethylethanolamine or dimethylethanolamine (but not serine, glycine, dimethylglycine, methylamine, hydroxylamine or $\beta$-hydroxyethylhydrazine) to the medium also resulted in appreciable increases in the colony radial growth rates of $A 3 / 5$ (increased by about $130 \%$ for choline) and $\mathrm{C} 106$ (increased by about $25 \%$ for choline). No significant difference was observed between the phospholipid compositions of the two strains, and the addition of $100 \mu \mathrm{M}$ choline to the medium had no significant effect on the phospholipid composition of either strain.
\end{abstract}

\section{INTRODUCTION}

Mycoprotein is a protein- and fibre-rich food for human consumption made from Fusarium graminearum (Edelman et al., 1983). Because of the contribution of morphology to the texture of mycoprotein, there is considerable interest in factors which affect the branching of mycelia of $F$. graminearum. Steele \& Trinci (1975) showed that the mean rate of extension $(E)$ of hyphae in a fungal mycelium is a function of the hyphal growth unit length ( $G$, the average length of the hypha associated with each hyphal tip) of the mycelium and its specific growth rate $(\mu)$. Thus,

$$
E=G \mu
$$

Equation (1) predicts that, provided $\mu$ remains constant, $E$ will be directly related to $G$. Thus, factors which reduce $E$ without affecting $\mu$ will result in the formation of mycelia which branch more profusely than untreated mycelia. Jejelowo \& Trinci (1988) have demonstrated a direct relationship between $G$ and colony radial growth rate $\left(K_{\mathrm{r}}\right)$, and therefore factors which cause a reduction in $G$ would be expected to cause a decrease in $K_{\mathrm{r}}$. Validamycin A and L-sorbose have no effect on $\mu$ but cause a decrease in $E$ and therefore in $G$ (Trinci, 1984; Robson et al., 1988, 1989). These compounds also cause a decrease in $K_{\mathrm{r}}$. Compounds which increase $E$ without affecting $\mu$ have not yet been identified. Such compounds would cause mycelia to branch more phosphatidylethanolamine; PI, phosphatidylinositol; PS, phosphatidylserine. 
sparsely than control mycelia (equation 1) and would result in an increase in $K_{\mathrm{r}}$. The observation of Strange \& Smith (1978) that choline and betaine increase the rate of extension of hyphae of $F$. graminearum, and that of Anderson \& Solomons (1984) that ' . . in batch culture, individual hyphal networks (of $F$. graminearum) have longer interbranch distances when choline is present in the medium ...' are therefore of considerable interest.

The purpose of the present study was to determine the effect of choline on the morphology $(G)$ and growth kinetics $\left(\mu, E\right.$ and $K_{\mathrm{r}}$ ) of the $F$. graminearum wild-type strain (A3/5) used in mycoprotein production, as well as on a colonial variant $(\mathrm{C} 106)$ which arose spontaneously during cultivation of the wild-type. The $\mathrm{C} 106$ variant branches more profusely than $\mathrm{A} 3 / 5$ and forms dense colonies on agar which expand in radius more slowly than wild-type colonies.

\section{METHODS}

Organism and media. F. graminearum Schwabe strains A3/5 and C106 were obtained from Mr T. W. Naylor, Marlow Foods, Billingham, UK. C106 is a variant which arose spontaneously during the prolonged growth of the wild-type strain (A3/5) in continuous culture. In our studies, the defined medium of Vogel (1956) was used with glucose $\left(10 \mathrm{~g}^{-1}\right)$ as the carbon source instead of sucrose. Vogel's mineral salts solution was prepared at $\times 50$ final concentration, sterilized by membrane $(0.2 \mu \mathrm{m}$ diameter) filtration and added to the glucose solution, which was sterilized by autoclaving at $121^{\circ} \mathrm{C}$ for $15 \mathrm{~min}$. For some experiments, the medium was supplemented with ethanolamine, monomethylethanolamine (MME), dimethylethanolamine (DME), choline, betaine, serine, glycine, dimethylglycine, methylamine, hydroxylamine or $\beta$-hydroxyethylhydrazine. These components were sterilized by membrane filtration and were added to the sterile glucose solution. For some experiments the medium was solidified with agar (Davis Gelatine; $15 \mathrm{~g}^{-1}$, final concentration).

Cultural conditions. Liquid batch cultures of $F$. graminearum were grown in $20 \mathrm{ml}$ volumes of medium in $250 \mathrm{ml}$ Nephlos flasks (Trinci, 1972). Each flask was inoculated with $1 \mathrm{ml}$ of a suspension of macroconidia in distilled water prepared by harvesting spores from 7- to 10-d-old cultures grown on agar solidified Vogel's medium; the macroconidia used for inocula were washed with $10 \mathrm{ml}$ sterile distilled water. The flasks were incubated on a rotary shaker (with a throw of $2.5 \mathrm{~cm}$ ) at 200 r.p.m.

Germlings of $F$. graminearum were grown in $9 \mathrm{~cm}$ diameter Petri dishes containing $20 \mathrm{ml}$ agar medium overlaid with Cellophane (PT 300, British Cellophane Ltd). Prior to use, the Cellophane was twice boiled for $15 \mathrm{~min}$ in distilled water to remove plasticizers. Cellophane-overlaid medium was inoculated with about 50 macroconidia, which were spread over the surface using a glass spreader; the Cellophane ensured that the mycelia grew in a single plane and could be photographed. Colonies of $F$. graminearum were grown in $9 \mathrm{~cm}$ diameter Petri dishes containing $20 \mathrm{ml}$ agar media which were inoculated centrally with $1.5 \mu \mathrm{l}$ of a suspension of macroconidia $\left(1 \times 10^{6}\right.$ spores $\mathrm{ml}^{-1}$ ). All cultures were incubated at $25^{\circ} \mathrm{C}$.

Measurements of fungal growth and morphology. Growth $(\mu)$ of liquid cultures was measured by increase in optical density using a colorimeter (Evans Electroselinium) with a green $(540-560 \mathrm{~nm})$ filter. Growth $(\mu$ and $E)$ of germlings on agar medium was determined by timelapse photomicroscopy as described by Trinci (1974). $E$ was calculated from

$$
E=\frac{2\left(H_{t}-H_{0}\right)}{B_{0}+B_{t}}
$$

where $H_{0}=$ total hyphal length at zero time, $H_{t}=$ total hyphal length $1 \mathrm{~h}$ later, $B_{0}=$ no. of tips at zero time, and $B_{t}=$ no. of tips $1 \mathrm{~h}$ later. Measurements of colony diameters were made with a rule at magnifications of $\times 10$ using a Shadowmaster (Baty \& Co.). Measurements of hyphal growth unit lengths were made on mycelia which had been grown for 17 to $26 \mathrm{~h}$ on agar medium or for 20 to $23 \mathrm{~h}$ in liquid medium. Mycelia with five or more hyphal tips were photographed at magnifications of $\times 40(\mathrm{~A} 3 / 5)$ or $\times 100(\mathrm{C106})$ and were then measured at a magnification of $\times 10$ using a Shadowmaster. Hyphal diameters were measured at a magnification of $\times 1000$ using an Olympus travelling micrometer eyepiece.

Phospholipid analysis. Lipids were extracted from fungal biomass following the procedure of Angus \& Lester (1972). The mycelium was grown at $25^{\circ} \mathrm{C}$ in $20 \mathrm{ml}$ volumes of liquid medium in $250 \mathrm{ml}$ Erlenmeyer flasks with or without $100 \mu \mathrm{M}$-choline, on a rotary shaker at 200 r.p.m. Cultures were harvested in late exponential phase by filtration through one layer of muslin. Phospholipids were extracted three times for $15 \mathrm{~min}$ at $60^{\circ} \mathrm{C}$ in $10 \mathrm{ml}$ solvent $\left(95 \%\right.$ aqueous ethanol/ $\mathrm{H}_{2} \mathrm{O} /$ diethylether/pyridine/concentrated ammonia, $15: 15: 5: 1: 0.018$, by vol.). The three extracts were combined and dried in a rotary evaporator at $45^{\circ} \mathrm{C}$. The lipid residue was dissolved in chloroform/methanol $(2: 1, \mathrm{v} / \mathrm{v})$, taken to dryness under a stream of nitrogen and stored at $-20^{\circ} \mathrm{C}$. The phospholipid extracts were dissolved in $0.5 \mathrm{ml}$ chloroform/methanol $(2: 1, \mathrm{v} / \mathrm{v})$ and $100 \mu \mathrm{l}$ was applied as a sr spot to activated thin-layer chromatography plates (Merck, silica gel G, $20 \times 10 \mathrm{~cm}$ ). The plates were develop $\epsilon$ in two dimensions with the following solvents: (1) chloroform/methanol/ammonia/ $\mathrm{H}_{2} \mathrm{O}(66: 27: 3: 0 \cdot 9 \mathrm{by}$ vol.) tor $1-$ $2 \mathrm{~h}$, and (2) chloroform/methanol/acetic acid/ $\mathrm{H}_{2} \mathrm{O}(32: 10: 5: 1$ by vol.) for 20-30 min, drying the plates between 
Table 1. Effect of choline on the specific growth rate, mean hyphal extension rate and hyphal growth unit length of mycelia of $F$. graminearum A3/5 and C106 cultured at $25^{\circ} \mathrm{C}$ on Vogel's agar medium

The results are means of 4 to $9\left(^{*}\right), 14$ to $42(\dagger)$ and 24 to $50(\ddagger)$ replicates, respectively, and are shown \pm standard error. Figures in the same column with the same superscript letter are not significantly different $(P>0.05)$.

\begin{tabular}{|c|c|c|c|c|}
\hline Strain & $\begin{array}{c}\text { Choline } \\
\text { conen } \\
(\mu \mathrm{M})\end{array}$ & $\begin{array}{c}\text { Specific } \\
\text { growth } \\
\text { rate }^{*} \\
\left(\mu, \mathrm{h}^{-1}\right)\end{array}$ & $\begin{array}{c}\text { Mean hyphal } \\
\text { extension } \\
\text { rate } \dagger \\
\left(E, \mu \mathrm{m} \text { per tip } \mathrm{h}^{-1}\right)\end{array}$ & $\begin{array}{c}\text { Hyphal growth } \\
\text { unit } \\
\text { length } \ddagger \\
(G, \mu \mathrm{m})\end{array}$ \\
\hline $\mathrm{A} 3 / 5$ & $\begin{array}{l}0 \\
1 \\
5\end{array}$ & $\begin{array}{l}0.28 \pm 0.01^{a} \\
0 \cdot 25 \pm 0 \cdot 01^{a} \\
0.26 \pm 0.02^{a}\end{array}$ & $\begin{array}{r}89 \pm 4^{a} \\
126 \pm 5^{b} \\
136 \pm 3^{b}\end{array}$ & $\begin{array}{l}323 \pm 10^{a} \\
515 \pm 17^{b} \\
513 \pm 12^{b}\end{array}$ \\
\hline $\mathrm{Cl} 106$ & $\begin{array}{c}0 \\
0 \cdot 1 \\
10 \\
1500\end{array}$ & $\begin{array}{l}0.28 \pm 0.01^{a} \\
0.26 \pm 0.01^{a} \\
0.26 \pm 0.01^{a} \\
0.31 \pm 0.01^{a}\end{array}$ & $\begin{array}{l}21 \pm 1^{c} \\
19 \pm 1^{c} \\
18 \pm 1^{c} \\
33 \pm 1^{d}\end{array}$ & $\begin{array}{r}69 \pm 2^{c} \\
65 \pm 3^{c} \\
63 \pm 3^{c} \\
104 \pm 3^{d}\end{array}$ \\
\hline
\end{tabular}

solvents to remove the ammonia. Phospholipids were visualized with a phosphomolybdate spray reagent (Dittmer \& Lester, 1964). The spots were marked and scraped into separate test tubes. Phosphate content was measured using the method of Rouser et al. (1970). The data were analysed for significant difference using Scheffe's multiple range test (Hicks, 1982).

\section{RESULTS}

Effect of choline on the growth and morphology of F. graminearum A3/5 and C106 cultured in agar medium

Table 1 shows that there was no significant difference $(P>0.05)$ between the specific growth rates of $\mathrm{A} 3 / 5$ and $\mathrm{C} 106$ cultured on agar medium. Further, addition of choline to the medium had no significant effect $(P>0.05)$ on the specific growth rate of either strain (Table 1$)$. Similarly, the mean diameter of hyphae of A3/5 was not affected by $5 \mu \mathrm{M}$-choline in the medium; hyphae had diameters of $4.7 \pm 0.1 \mu \mathrm{m}$ and $4.8 \pm 0.1 \mu \mathrm{m}$ in the absence and presence of $5 \mu \mathrm{M}-$ choline, respectively. However, the mean hyphal extension rate and hyphal growth unit length of mycelia of A3/5 were significantly increased $(P<0.05)$ by the addition of as little as $1 \mu \mathrm{M}$ choline to the medium (Table 1). By contrast, the length of the hyphal growth unit and mean hyphal extension rate of $\mathrm{C} 106$ were significantly $(P<0.05)$ increased only by a choline concentration of $1.5 \mathrm{~mm}$ (Table 1).

\section{Effect of choline on the growth and morphology of F. graminearum A3/5 and C106 in shake flask culture}

Because L-sorbose affects the branching $(G)$ of mycelia of Neurospora crassa cultured on agar but not on liquid medium (Trinci \& Collinge, 1973), it cannot be assumed that the effects of choline on hyphal extension and branching of mycelia of $F$. graminearum cultured on agar medium would also be observed in liquid culture. However, the results in Table 2 confirm that, as on agar media, mycelia of $\mathrm{A} 3 / 5$ and $\mathrm{C} 106$ cultured in liquid media branch more sparsely when the medium contained choline. Both strains had the same specific growth rate $\left(0.24 \pm 0.004 \mathrm{~h}^{-1}\right.$; mean of six replicates \pm standard error) in shake flask culture and specific growth rate was not affected by concentrations of choline of up to $1 \mathrm{mM}$ (results not shown).

If it is assumed that the volume of the hyphal growth unit $\left(G_{\mathrm{v}}\right)$ remains constant during mycelial growth, it follows that a relatively small change in hyphal radius will have an appreciable effect on hyphal growth unit length, since, as shown below, $G$ is inversely related to the square of hyphal radius ( $r$ ) (Trinci, 1984).

$$
G=\frac{G_{\mathrm{v}}}{\pi r^{2}}
$$


Table 2. Effect of choline on the growth and morphology of mycelia of $F$. graminearum A3/5 and C106 cultured at $25^{\circ} \mathrm{C}$ on Vogel's liquid medium in shake flask culture

The results are means of at least $13\left(^{*}\right)$ and $20(\dagger)$ replicates, respectively, and are shown \pm standard error. Figures in the same column with the same superscript letter are not significantly different $(P>0.05)$.

$\begin{array}{lcccc}\text { Strain } & \begin{array}{c}\text { Choline } \\ \text { concn } \\ (\mu \mathrm{M})\end{array} & \begin{array}{c}\text { Hyphal growth } \\ \text { unit length* } \\ (G, \mu \mathrm{m})\end{array} & \begin{array}{c}\text { Mean hyphal } \\ \text { diametert } \\ (\mu \mathrm{m})\end{array} & \begin{array}{c}\text { Hyphal growth } \\ \text { unit volume } \\ \left(G_{\mathrm{v}}, \mu \mathrm{m}^{3} \times 10^{-3}\right)\end{array} \\ \mathrm{A} 3 / 5 & 0 & 276 \pm 11^{a} & 4 \cdot 2 \pm 0 \cdot 1^{a b} & 3.77 \\ & 1 & 293 \pm 11^{a} & 3 \cdot 7 \pm 0 \cdot 1^{b} & 3 \cdot 20 \\ & 10 & 491 \pm 20^{b} & 4 \cdot 4 \pm 0 \cdot 1^{a c} & 7 \cdot 30 \\ \text { C106 } & 100 & 449 \pm 18^{b} & 4 \cdot 4 \pm 0 \cdot 1^{a c} & 6 \cdot 70 \\ & 1000 & 478 \pm 20^{b} & 4 \cdot 3 \pm 0 \cdot 1^{a b c} & 6 \cdot 78 \\ & 0 & 90 \pm 2^{c} & 4 \cdot 6 \pm 0 \cdot 1^{a c} & 1 \cdot 50 \\ & 1 & 91 \pm 3^{c} & 4 \cdot 4 \pm 0 \cdot 1^{a c} & 1 \cdot 39 \\ & 10 & 103 \pm 5^{c} & 4 \cdot 6 \pm 0 \cdot 1^{a c} & 1 \cdot 70 \\ & 100 & 96 \pm 4^{c} & 4 \cdot 8 \pm 0 \cdot 1^{c} & 1 \cdot 74 \\ & 1000 & 139 \pm 17^{d} & \text { ND } & \text { ND }\end{array}$

Table 2 shows that mycelia of A3/5 and C106 differed in hyphal growth unit length and hyphal growth unit volume, but not in hyphal diameter. Thus, the difference observed in hyphal growth unit length between $\mathrm{A} 3 / 5$ and $\mathrm{Cl06}$ (Table 1) cannot be explained in terms of a change in hyphal radius. The length of the hyphal growth units of $\mathrm{A} 3 / 5$ and $\mathrm{C106}$ in submerged culture were significantly $(P<0.05)$ increased at choline concentrations of $10 \mu \mathrm{M}$ and $1 \mathrm{mM}$, respectively.

Effect of betaine, choline and precursors of choline on the radial growth rates of colonies of F. graminearum A3/5 and C106

When grown on Vogel's agar medium lacking choline, $F$. graminearum A3/5 and C106 had colony radial growth rates of $144 \pm 2$ (mean \pm standard error) and $57 \pm 1 \mu \mathrm{m} \mathrm{h}^{-1}$ respectively. Fig. 1 shows the effect of choline concentration on the radial growth rate of $A 3 / 5$; an increase in colony radial growth rate of $130 \%$ (difference between the radial growth rates of treated and untreated colonies, expressed as a percentage of the radial growth rate of untreated colonies) was observed at a choline concentration of $5 \mu \mathrm{M}$. The relationship between $K_{\mathrm{r}}$ and $G$ in A3/5 is not linear and maximum $G$ values were observed at $1 \mu \mathrm{M}$-choline (Table 1). The radial growth rate of $\mathrm{C} 106$ was increased by $25 \%$ when colonies were grown on medium containing $100 \mu \mathrm{M}$-choline. No further increase in $K_{\mathrm{r}}$ was observed when the concentration of choline in the medium was increased to $2 \mathrm{mM}$.

Fig. 1 also shows the effect of betaine and various precursors of choline (ethanolamine, MME and DME) on the radial growth rate of colonies of $\mathrm{A} 3 / 5$. Like choline, the three precursors of choline tested increased colony radial growth rate by almost $130 \%$, but betaine caused an increase in colony radial growth rate of only $105 \%$. For C106, MME and betaine gave approximately the same increase in colony radial growth rate as choline, but DME (increase of $40 \%$ ) and ethanolamine (increase of $16 \%$ ) gave increases which were greater and lower, respectively, than the increases observed for choline (results not shown). The $K_{\mathrm{r}}$ of A3/5 or C106 did not increase with $100 \mu \mathrm{M}$ concentrations of serine, glycine, dimethylglycine, methylamine, hydroxylamine or $\beta$-hydroxyethylhydrazine (results not shown).

\section{Phospholipid composition of A3/5 and C106}

Increased branching of Rhizoctonia cerealis has been correlated with a reduced phosphatidylinositol content (Robson et al., 1989), and the in vitro activities of chitin synthase and chitinase (enzymes involved in wall growth and branching) are stimulated by phosphatidylcholine (PC) (Humphreys \& Gooday, 1984; Vermeulen \& Wessels, 1983). The latter results suggest that the PC content of the protoplasmic membrane of fungi may affect the activity of membrane-bound 


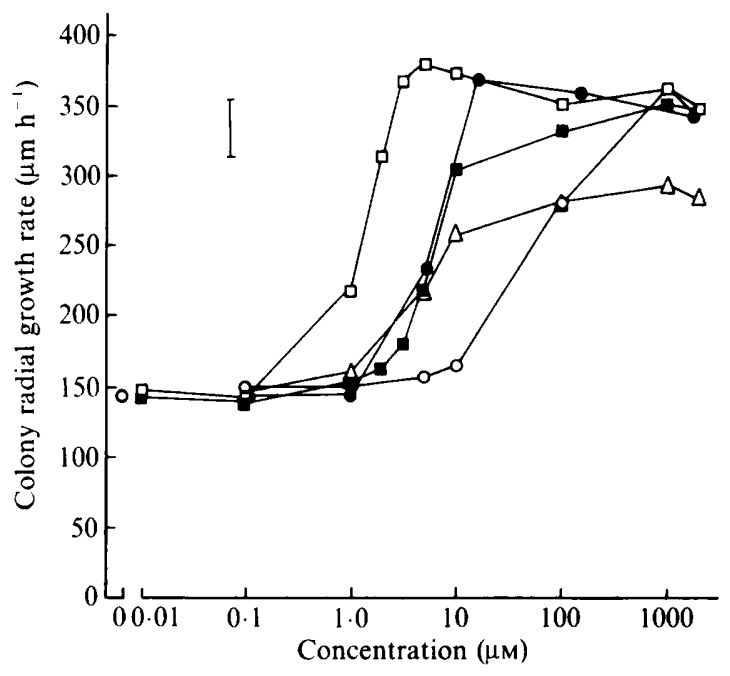

Fig. 1. Effects of choline $(\square)$, DME $(\square), \operatorname{MME}(\bigcirc)$, ethanolamine $(O)$ and betaine $(\triangle)$ concentration on the radial growth rate of $F$. graminearum A3/5. The error bar represents $95 \%$ confidence interval for sample with greatest variation (ten replicates per treatment).

enzymes involved in synthesis of the hyphal wall. Thus, choline might alter branching of $F$. graminearum because of its effect on the PC content of the biomass.

The results in Table 3 show that the main phospholipids of $F$. graminearum were PC, phosphatidylethanolamine (PE), phosphatidylinositol (PI), and phosphatidylserine (PS). No significant difference was observed between the phospholipid compositions of the two strains, and addition of $100 \mu \mathrm{M}$-choline to the medium had no significant effect on the phospholipid composition of either strain.

\section{DISCUSSION}

The present results confirm the validity of equation (1), as mean hyphal extension rates estimated from $\mu$ and $G$ are very similar to the experimental values observed for $E$ (Table 1). An important result is that, for the first time, a compound (choline) has been identified which increases mean hyphal extension rate and hyphal growth unit length, without affecting specific growth rate (Table 1). Increases in $E$ and $G$ caused by choline are correlated with increases in hyphal growth unit volume in the absence of a change in hyphal diameter (Table 1). Thus, mean hyphal extension rate of $F$. graminearum is increased because, in the presence of choline, tip growth is supported by an increased volume of protoplasm. Similarly, the profuse branching of C106 compared with the parental strain is correlated with decreases in hyphal growth unit volume and mean hyphal extension rate (Table 1).

The present results suggest that the increased radial growth rate of colonies of $F$.graminearum in the presence of choline observed by Strange et al. (1974), Anderson \& Solomons (1984) and in Fig. 1 , is not due to a change in specific growth rate but (indirectly) to the change observed in branching $(G)$.

Strange \& Smith (1978) suggested two possible explanations for the effect of choline on $F$. graminearum, viz. increased incorporation of choline into phospholipid, or increased methylation power. The predominant pathway of PC synthesis in most fungi is the BremerGreenberg methylation pathway, in which PC is synthesized by the succesive methylation of PE (Hubbard \& Brody, 1975; Markham \& Bainbridge, 1979). PC may also be synthesized directly from choline via the Kennedy cytidine nucleotide pathway (Kennedy \& Weiss, 1956; Matysiak et al., 1974; Wilson \& Barran, 1983). Unless there is a metabolic block in the pathway of choline synthesis, precursors of PC should have effects similar to choline. 


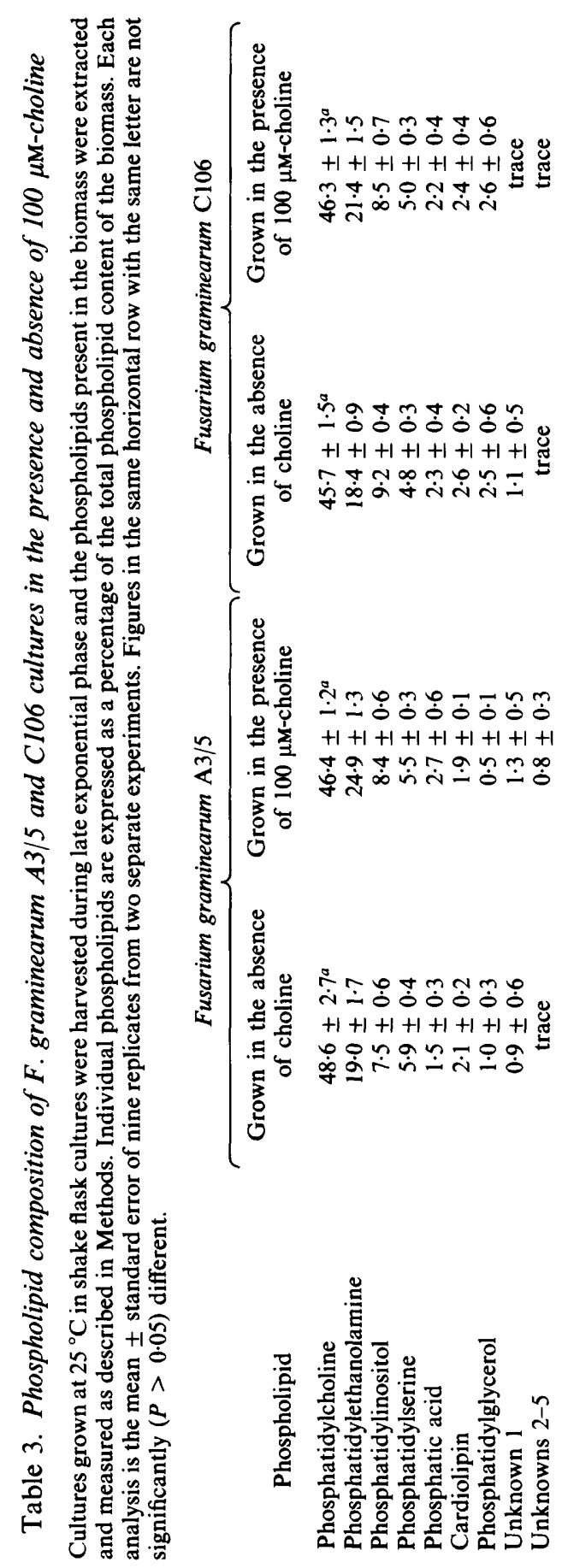


PE, MME and DME, like choline, caused a $130 \%$ increase in colony radial growth rate of A3/5. As the choline precursors utilize methylating power in the synthesis of choline, their effectiveness in stimulating colony radial growth rate (Fig. 1) suggests that exogenous choline does not cause its effects via an increase in methylating power. Betaine is the oxidative product of choline (Jukes, 1947). It was as effective as choline in increasing the colony radial growth rate of $\mathrm{C} 106$, but was less effective than choline in increasing the colony radial growth rate of $\mathrm{A} 3 / 5$ (Fig. 1). As betaine is the immediate donor of methyl groups to methionine, its inability to stimulate the radial growth rate of colonies of $\mathrm{A} 3 / 5$ as effectively as choline suggests that choline and its precursors do not affect $F$. graminearum because of increased methylating power.

The present results show that PC levels of $F$. graminearum are not increased when $100 \mu \mathrm{M}$ choline is added to the medium (Table 3). Therefore, the effect of choline on branching of $F$. graminearum cannot be explained in terms of a changed membrane phospholipid composition affecting the activity of membrane-bound enzymes such as chitin synthase. Further, the hypothesis that exogenous or endogenous free-choline increases the activity of protoplasmic membrane associated enzymes also seems unlikely to be valid as the choline precursors, ethanolamine, MME and DME, and the choline oxidation product, betaine, cause similar effects, suggesting that choline metabolism is involved in the response in some unknown way. It is possible that these compounds act by inducing certain enzymes. Methylamine, hydroxylamine and $\beta$-hydroxyethylhydrazine (amines) did not stimulate $K_{\mathrm{r}}$, nor did serine, a precursor of ethanolamine, or glycine and dimethylglycine, the oxidation products of betaine. These results show that the choline effect is not a general amine effect but is instead confined to choline, betaine and their immediate precursors. Thus, although the present results show that concentrations of choline as low as $1 \mu \mathrm{M}$ (Table 1) alter branching and extension of hyphae of $F$. graminearum, no satisfactory hypothesis can be advanced to explain this effect.

We thank the Natural Science and Engineering Research Council of Canada and Marlow Foods for supporting this research.

\section{REFERENCES}

Anderson, C. \& Solomons, G. L. (1984). Primary metabolism and biomass production from Fusarium. In The Applied Mycology of Fusarium, pp. 231-250. Edited by M. O. Moss \& J. E. Smith. London: British Mycological Society.

ANGus, W. W. \& Lester, R. L. (1972). Turnover of inositol and phosphorus containing lipids in Saccharomyces cerevisiae; extracellular accumulation of glycerophosphatidylinositol deriwed from phosphatidylinositol. Archives of Biochemistry and Biophysics. 151, 483-495.

DitTMER, J. C. \& Lester, R. L. (1964). A simple specific spray reagent for the detection of phospholipids on thin-layer chromatograms. Journal of Lipid Research 5, 126-127.

Edelman, J., Fewell, A. \& Solomons, G. L. (1983). Myco-protein - a new food. Nutrition Abstracts and Reviews 53, 471-480.

Hicks, C. R. (1982). Fundamental Concepts in the Design of Experiments. New York: Holt, Reinhart and Winston.

HubBard, S. C. \& Brody, S. (1975). Glycerophospholipid variation in choline and inositol auxotrophs of Neurospora crassa. Journal of Biological Chemistry 250, 7173-7183.

Humphreys, A. M. \& GOODAY, G. W. (1984). Phospholipid requirement of microsomal chitinase from Mucor mucedo. Current Microbiology 11, 187190.
Jejelowo, O. A. \& TrincI, A. P. J. (1988). Effect of the paramorphogens 3-O-methylglucose, glucosamine and sorbose on growth and morphology of Botrytis fabae. Transactions of the British Mycological Society 91, 633-660.

Jukes, T. H. (1947). Choline. Annual Review of Biochemistry 16, 193-222.

KENNEDY, E. P. \& WEISS, S. B. (1956). The function of cytidine co-enzymes in the biosynthesis of phospholipids. Journal of Biological Chemistry 222, 193-214.

MARKHAM, P. \& BAINBRIDGE, B. W. (1979). Characterization of a new choline locus in Aspergillus nidulans and its significance for choline metabolism. Genetical Research 32, 303-310.

MATYSIAK, Z., RADOMINSKA-PYREK, A. \& CHOJNACKI, T. (1974). The cytidine mechanism and methylation pathway in the formation of $N$-methylated ethanolamine phosphoglycerides in Neurospora crassa. Molecular and Cellular Biochemistry 3, 143151.

Robson, G. D., Kuhn, P. J. \& Trinci, A. P. J. (1988). Effects of validamycin A on the morphology, growth and sporulation of Rhizoctonia cerealis, Fusarium culmorum and other fungi. Journal of General Microbiology 134, 3187-3194.

Robson, G. D., Kuhn, P. J. \& TRINCI, A. P. J. (1989). Effect of validamycin A on the inositol content and branching of Rhizoctonia cerealis and other fungi. Journal of General Microbiology 135, 739-750. 
Rouser, G., Fleischer, S. \& Yamamoto, A. (1970). Two dimensional thin layer chromatographic separation of polar lipids and determination of phospholipids by phosphorus analysis of spots. Lipids $\mathbf{5}$, 494-496.

Steele, G. C. \& Trinci, A. P. J. (1975). Morphology and growth kinetics of hyphae of differentiated and undifferentiated mycelia of Neurospora crassa. Journal of General Microbiology 91, 362-368.

Strange, R. N. \& SMIth, H. "(1978). Specificity of choline and betaine as stimulants of Fusarium graminearum. Transactions of the British Mycological Society 70, 187-192.

Strange, R. N., Majer, J. R. \& Smith, H. (1974). The isolation and identification of choline and betaine as the two major components in anthers and wheat germ that stimulate Fusarium graminearum in vitro. Physiological Plant Pathology 4, 277-290.

TRINCI, A. P. J. (1972). Culture turbidity as a parameter of mould growth: Transactions of the British Mycological Society 58; 467-473.

TrINCI, A. P. J. (1974). A study of the kinetics of hyphal extension and branch initiation of fungal mycelia. Journal of General Microbiology 81, 225236.

TrINCI, A. P. J. (1984). Antifungal agents which affect hyphal extension and hyphal branching. In Mode of Action of Antifungal Agents, pp. 113-134. Edited by A. P. J. Trinci \& J. F. Ryley. London: British Mycological Society.

Trinci, A. P. J. \& Collinge, A. (1973). Influence of Lsorbose on growth and morphology of Neurospora crassa. Journal of General Microbiology 78, 179-192.

Vermeulen, C. A. \& Wessels, J. G. H. (1983). Evidence for a phospholipid requirement of chitin synthase in Schizophyllum commune. Current Microbiology 8, 67-71.

VoGEL, H. J. (1956). A convenient growth medium for Neurospora (Medium N). Microbial Genetics Bulletin 13, 42-44.

Wilson, A. C. \& Barran, L. R. (1983). Effect of temperature on phosphatidylcholine synthesis by hyphae of Fusarium oxysporum f.sp. lycopersici. Transactions of the British Mycological Society 80, 113-116. 